\title{
Algunas reflexiones en torno a la 'teoría de los campos' y su contribución para el análisis de la universidad en el contexto de las actuales políticas de educación superior
}

\author{
Some reflections on 'Field Theory' and its contribution to \\ analysis of the university in the context of current policies on \\ higher education
}

HÉCTOR MORA NAWRATH Universidad Católica de Temuco, Chile

RECEPCIÓN: I7/O8/20I3·ACEPTACIÓN: 20/IO/20I3

RESUMEN Muchos especialistas han reflexionado e investigado el proceso de cambio y actual situación de la educación superior universitaria en Latinoamérica y el mundo. Existe coincidencia en que estos cambios poseen un carácter global, exhibiendo desde comienzos de los ochenta una tendencia heteronomizante en el marco de un 'Estado evaluador' que busca mejorar la gestión económica y calidad universitaria, incorporando nuevos actores como empresa privada y con ello una lógica de mercado. Así, se han implementando políticas de educación superior que buscan aumentar la productividad y la calidad por medio de mecanismos de regulación que han llevado o llevarán a nuevas configuraciones del trabajo académico y la organización universitaria. En este contexto, este trabajo busca establecer una reflexión proponiendo como base la 'teoría de los campos' propuesta por Bourdieu, con el objeto de visualizar cómo ésta permite pensar nuevas configuraciones, así como anticipar escenarios posibles en el marco de la lucha por la apropiación de distintos capitales que actualmente se están poniendo en juego en distintas escalas: entre universidades, entre estamentos universitarios, entre facultades y entre académicos. 
PALABRAS CLAVE Educación superior universitaria, políticas de educación superior, teoría de los campos.

ABSTRACT Many specialists have considered and conducted research on changes in, and the current situation of, higher education within universities both in Latin America and the world. Coincidentally, these changes have a global character. There has been a heteronomizing trend since the rg8os in terms of a framework of an 'Evaluating State' which seeks to improve the economic management and quality of universities by incorporating new actors such as private companies and, hence, the logic of the market. Thus higher education policies have been implemented which seek to increase productivity and quality through regulatory mechanisms that will lead to new forms of academic activity and university organization. In this context, and on the basis of Bourdieu's Field Theory, this article examines how his theory allows us to think about these new forms, and to foresee possible scenarios in the framework of the struggle to appropriate the various issues which are currently in play on different levels: between universities, between university bodies, between faculties and between academics.

KEYWORDS University higher education, higher education policies, field theory.

\section{A modo de introducción: un diagnóstico de la situación actual}

Existe acuerdo en que la universidad ha experimentado sucesivas transformaciones a través de la historia, transformaciones que han tenido como trasfondo diversas situaciones sociopolíticas, cuya expresión se ha manifestado en reformas localizadas en contextos específicos vinculadas a la reproducción de distintos modelos de universidad (Ben-David y Zloczower, I966; Drèze y Debelle, 2008; Clark, I992; Altbach, 200I; Krotsch, 2009; Tünnermann, 2003). Sin embargo, a partir de los años ochenta dichas transformaciones, a pesar de mantener cierta especificidad local (Brunner, I990; Muñoz y Rodríguez, 2000; Altbach, 200I; Krotsch, 2009; García, 2003; Mollis, 2003; Chiroleu, 2007a; Marquina, 2007), ${ }^{1}$ adquieren un carácter más amplio, que deja de manifiesto

I. De hecho, la emergencia del mismo término 'educación superior' es un reflejo de éstas. Para Krotsch (2009), el término marca el paso de la universidad de élite a la universidad de masas, y con esto el incremento en la diferenciación de la oferta 
los efectos de una política internacional y/o transnacional que se entiende a la luz de la globalización, el modelo neoliberal y el nuevo rol que asume el Estado. Entre dichas tendencias podemos mencionar:

I. Masificación de la educación superior expresada en la expansión de la matrícula (Brunner, 2007; Schugurensky, I998; Altbach, 200I; Krotsch, 2009; García, 2003, 2006; López, 2003; Mollis, 2003; Rodríguez, 2003; Tünnermann, 2003; Ibarra, 2005).

2. Reducción del financiamiento estatal, con mayor control del gasto e inversión en el sector, lo que ha llevado a buscar otras fuentes presupuestales y a generar mecanismos de eficiencia administrativa (Schugurensky, I998; Krotsch, 2009; López, 2003; Mollis, 2003; Llomovatte, 2006), y el consiguiente desplazamiento del gobierno académico (Altbach, 200I; Marquina, 2007).

3. Presencia de inversión privada en la oferta de educación superior, asociada a procesos de privatización y mercantilización, con la consiguiente emergencia de nuevas ofertas educativas (Schugurensky, I998; Altbach, 200I; Krotsch, 2009; García, 2003, 2006; López, 2003; Mollis, 2003; Tünnermann, 2003; Llomovatte, 2006; Marquina, 2007; Rodríguez, 2003).

4. Alianzas estratégicas entre agencias internacionales y gobiernos, así como entre universidades, corporaciones y sector público (Alcántara, 2000; García, 2003, 2006; López, 2003; Llomovatte, 2006; Rodríguez, 2003).

5. Pérdida de exclusividad de la universidad como única instancia generadora de conocimiento. Visualizada como empresa de conocimiento, entra en el mercado por medio de la competitividad con otras instituciones no tradicionales en el área de la formación superior y la investigación (Brunner, 2007; Altbach, 2001; García, 2003, 2006; López, 2003; Mollis, 2003).

6. Políticas dirigidas a la regulaciones de educación superior promovidas por los gobiernos, y particularmente aquellas orientadas al «control de calidad» mediante procesos de evaluación y acreditación institucional,

universitaria (pública y privada), así como la complejización y diferenciación de instituciones. 
certificación de programas, etcétera, así como la creación organismos acreditadores y evaluadores (Schugurensky, I998; Krotsch, 2009; García, 2003, 2006; González, 2003; Mollis, 2003; Rodríguez, 2003; Araujo, 2007; Ibarra, 2007). ${ }^{2}$

7. Reformas académicas dirigidas a la reducción de los años de titulación, obtención de títulos intermedios, flexibilidad curricular, modelos educativos orientados a competencias profesionales (Mollis, 2003; Rodríguez, 2003; Tünnermann, 2003), lo que Schugurensky (I998) también ha denominado como vocacionalización.

8. Diferenciación académica en base a evaluación por productividad en el marco de jerarquización y categorización docente (Schugurensky, I998; Rodríguez, 2003; Prati y Prego, 2007), que se desprende del fenómeno de la profesionalización académica (Brunner, 2007; Altbach, 200I, 2003; Llomovatte, 2006; Marquina, 2007). ${ }^{3}$

9. Incorporación de nuevas tecnologías de información y comunicación, han generado nuevos escenarios tecnológicos que modifican los procesos a través de los cuales circula y se desarrolla la información y el conocimiento. Se generan instancias institucionales de formación a distancia (no presenciales), lo que se acompaña de la deslocalización y transnacionalización de la educación (Schugurensky, I998; García, 2003, 2006; Mollis, 2003; Rodríguez, 2003; Tünnermann, 2003).

Las reorientaciones en las políticas de educación superior, que se expresa en las tendencias antes descritas, tienen su origen en la crítica que el Estado y la sociedad realizan al rol desempeñado por las universidades. Ello decanta

2. Bajo la figura del consultor experto se canaliza la externalización de la evaluación de calidad que ha sido definida desde el Estado, y que radica en consultorías dirigidas a la evaluación universitaria y curricular (Marquina, 2009), pudiendo asumir dicha tarea un individuo o una organización acreditada para tales fines. Con ello se busca regular descompensando la carga que implicaría la evaluación por parte del Estado, intentando además brindar objetividad y transparencia a un proceso que requiere y apunta hacia la calidad.

3. Como señala Brunner ( 1985 ), este fenómeno obedece a la conformación de un mercado universitario, donde el trabajo académico se desarrolla bajo la forma de una relación salarial, convirtiéndose la universidad en un importante espacio ocupacional que se transforma en la meta de vastas capas de intelectuales. 
en una clara disposición al control y evaluación económica y de calidad de estas instituciones (Levy, I994; Brunner, I997; Schugurensky, 2000; Alcántara, 2000; Mollis, 2003.; Rodríguez y Alcántara, 2003; Tünnermann, 2003; Chiroleu y Iazzetta, 2005; Llomovatte, 2006; Chiroleu, 2007a), ${ }^{4}$ que sería expresión de otro fenómeno global, la 'metamorfosis' que vive el Estado. El paso de un 'Estado protector' a un 'Estado evaluador' (Brunner, I990; Araujo, 2007; Chiroleu y Iazzetta, 2005; Chiroleu, 2007a), trae consigo la demanda de estándares elevados de eficiencia, exigiendo conductas emprendedoras ligadas a la generación de recursos a través de innovaciones, así como la introducción del mercado como principal regulador. Chiroleu (2007a) señala que el 'Estado evaluador' genera una nueva distribución del poder que articula una retórica que reconoce la autonomía, y, al mismo tiempo, extrema la evaluación de resultados y calidad de proceso y servicios bajo estándares internacionales que mediatiza el financiamiento diferencial para las instituciones educativas en base a metas. El poder de decisión y la autonomía del campo académico, otrora en la esfera universitaria, se traslada hacia el Estado y en mayor medida hacia el mercado, lo que trae consigo nuevas configuraciones para el quehacer en el espacio universitario.

Para algunos especialistas, el nuevo escenario favorece el debate académico y político-administrativo en torno a la tensión entre autonomía/heteronomía, debate que sería constitutivo del nuevo 'espacio universitario' (Rinesi y Soprano, 2007), posibilitando la emergencia de propuestas en el marco de las innovaciones que las instituciones universitarias pueden realizar de manera de acoplarse al nuevo contexto (Llomovatte, 2006). Sin embargo, más allá de las oportunidades y desafíos, las dinámicas implicadas en este nuevo escenario pueden generar una serie de tensiones a distinto nivel, y profundizar la desigualdad y diferenciación que históricamente ha jugado en la definición y clasificación de las instituciones de educación superior en la distinción metrópoli-periferia. La rúbrica mercantilista y burocrática que envuelve el trabajo

4. Schugurensky (2000) señala que en el marco del Estado neoliberal y las dinámicas globalizadoras, la universidad es percibida como parte del problema económico ligado a la falta de competitividad internacional. En esta lógica, gobierno y empresa la conciben como una instancia que drena recursos públicos con escasa contribución a la generación de una economía competitiva, siendo los objetivos de autonomía institucional, crítica social y desarrollo cultural percibidos como obsoletos reductos de intereses particulares. 
académico (Marquina, 2007), y que tributa en exigencias evaluativas formalizadas del desempeño profesional e institucional — productividad en investigación, desarrollo de labores de extensión y mantención de calidad docente (Parati y Prego, 2007) - se refuerza a través de tecnologías que acompañan la evaluación, y que se objetivan en ranking de universidades que ubican a estas instituciones y a sus académicos en unas u otras posiciones. La jerarquización incentiva la disputa por posiciones, distinciones, recursos e incentivos conforme se cumplan metas asociadas a indicadores que regulan a cada plantel $-\mathrm{y}$ que poco a poco se estandarizan - y al sistema universitario en general, lo que propicia la acumulación y control del flujo de capital social, simbólico y económico no sólo entre académicos, sino también entre universidades.

De este modo, es posible observar la emergencia de una tendencia mercantilista, donde se fomenta la obtención de financiamientos privados e incorporan criterios de regulación de corte empresarial orientados por la maximización. Por otro lado, esta lógica se orienta a la paulatina privatización de instituciones públicas y desnacionalización de las privadas, con la subsecuente transnacionalización de una educación privatizada y mercantilizada (Altbach, 200I; Krotsch, 2009; Ibarra, 2005; Chiroleu, 2007). En el contexto de la transnacionalización de la educación, destacan el papel de los organismos y agencias internacionales ${ }^{5}$ y los burotecnócratas que instalan localmente temáticas y promueven las reformas (Alcántara, 2000; Rodríguez y Alcántara, 2003; Tünnermann, 2003; Llomovatte, 2006; Chiroleau, 2007; Chiroleu y Vilosio, 2007).

En síntesis, la crisis de hegemonía y legitimidad de la universidad que redunda en una crisis de institucionalidad (Krostch, 2007), desencadena una serie de adecuaciones internas con el fin de cumplir con las demandas externas o del entorno (Brunner, I997; Schugurensky, I998; Tünnermann, 2003; Altbach, 2008). Ello es expresión de la tendencia heteronomizante que afecta a la educación superior, la que emana de las nuevas características que adquiere el Estado, el mercado y la sociedad en un modelo económico neoliberal globalizado (Schugurensky, I998, 2000; Altbach, 2001, 2008; Krotsch, 2009; García, 2003, 2006; López, 2003; Ibarra, 2003; Llomovatte, 2006; Chiroleu, 2007a; Chiroleu y Vilosio, 2007; Llomovatte, 2006; Marquina, 2007; Mazzola, 2007; Rinesi y Soprano, 2007). Así, se plantea que la política universitaria

5. Entre ellas, el Fondo Monetario Internacional, Banco Interamericano de Desarrollo, Banco Mundial, Organización para la Cooperación y el Desarrollo Económico, Organización de las Naciones Unidas para la Educación, la Ciencia y la Cultura. 
se ha orientado explícitamente hacia las demandas del entorno, expresando una tendencia transicional desde un modelo de autonomía a un modelo heterónomo 'paradójico' (Schugurensky, I998), ${ }^{6}$ trascendiendo la clásica subordinación a «un grupo empresarial a través de donaciones o de la intervención coyuntural de un gobierno autoritario» (Schugurensky, 2000: II 7). ${ }^{7}$ Varios autores plantean que esto puede llevar al desgaste de valores y tradiciones ligados a la misión crítica y social de la universidad, la autonomía institucional y libertad académica, el logro de mayor acceso y equidad, y la generación de conocimiento como máxima orientadora (Schugurensky, I998; Altbach, 200 I; Mollis, 2003; Araujo, 2007)..$^{8}$

\section{Una revisión de la teoría del campo}

El análisis de la educación superior ha contribuido a identificar distintas dinámicas a nivel local y global, describir transformaciones históricas, realizar diagnósticos de la situación actual y visualizar ciertas propuestas para hacer frente o adaptarse a dichas transformaciones. Estas contribuciones provienen de aproximaciones muy diversas, ${ }^{9}$ situándose desde diferentes niveles o escalas

6. Plantea que el modelo heterónomo es paradójico, en el sentido que los gobiernos demandan contar con fuentes privadas de ingreso y en tanto las demandas se justifican en el aumento de la autonomía institucional y se endurecen los controles sobre los resultados.

7. Para Schugurensky (2000), «en el modelo heterónomo, el poder para definir la misión, la agenda y los productos de las universidades reside cada vez más en agencias externas y cada vez menos en sus propios órganos de gobierno. Mientras el principio de autonomía sugiere la capacidad de autodeterminación, independencia y libertad, el concepto de heteronomía se refiere a la subordinación a un orden impuesto por agentes externos» (II6-II7).

8. Rinesi y Soprano (2007) se manifiestan críticos en este aspecto. Para ellos, los académicos suelen identificar sus valores y posiciones como las que deberían ser sostenidas por la universidad, lo que representa una visión que se deriva de la posición en la trama universitaria. Estas formas de hegemonizar el discurso y el debate representarían «un factor de heteronomía tan grande y tan grave como los otros...» (22). Sentencian que «la autonomía universitaria, la autonomía de la universidad como la cosa pública, no puede confundirse con la afirmación excluyente de ningún interés o grupo de intereses particulares» (22).

9. Krotsch identifica el modelo analítico de Durkheim, Clark y Bourdieu. En térmi- 
de análisis - macro, micro, internistas, externistas, comparativos o casosy/o remitiéndose a una dimensión del complejo sistema que conforma la educación superior —disciplina, profesión académica, evaluación institucional, etcétera (Ben-David y Zloczower, I966; Clark, I992; Bourdieu, 2008; Brunner, 2007; Altbach, 200I, 2003; Schugurensky, I998; Becher, 200I).

$\mathrm{Al}$ respecto, si bien existen puntos concordantes, no se articula una visión única sobre el proceso y repercusiones que genera el nuevo escenario en torno al sistema de educación superior. En este plano, podemos hablar de una 'disputa por lo que está en juego' o una disputa de sentido en torno a la representación del objeto, que involucra a estudiantes-clientes, investigadores especialistas, profesionales académicos, autoridades y gobierno universitario, los órganos reguladores del Estado, la empresa, agencias multilaterales, las instituciones bancarias. Por otro lado, si bien es posible definir diferentes escalas de análisis - por ejemplo facultad, universidad, sistema de educación, etcétera-, se hace necesario entender cada componente implicado en la trama o red de relaciones; ${ }^{10}$ el carácter complejo que asume la educación superior, y con ella la universidad (Clark, I992), requiere una perspectiva relacional que supere abordajes exclusivamente internista o externista - universitario/ extrauniversitario- o centrados en los actores o en estructuras organizativas.

Una de las teorías que recoge precisamente estos dos núcleos analíticos es la 'teoría de los campos' propuesta por Bourdieu; parafraseando al autor, un análisis interno propondría una aproximación que concibe que los problemas que atañen y atraviesa la educación superior son generados por las propias instituciones educativas, en tanto un análisis externista atribuiría la aparición de ciertas problemática a las condiciones sociales. El concepto de campo (Bourdieu, I990; Bourdieu y Wacquant, 2005) es relacional, y su aplicación expresa potencial analítico en tanto la educación adquiere un talante mercantil en el cual se implican múltiples actores (Brunner, 2007) que pugnan por establecer

nos más generales, es posible encontrar perspectivas vinculadas a las corrientes funcionalista, estructuralista, sociocríticas, fenomenológicas, integracionistas, constructivistas, entre otras.

IO. Al respecto, podríamos preguntar ¿por qué algunos organismos gubernamentales en ciencia y tecnología validan y exigen publicaciones en determinados índex? ¿Por qué los rankings de universidades, los fondos que se asignan, las calificaciones y jerarquizaciones académicas consideran ciertos índex para la asignación de incentivos y recompensas? ¿Cuál es el origen de estos índex? ¿Qué capitales están involucrados? 
la reglas legítimas que deben operar y las exigencias a cumplir para seguir en juego (los estándares de calidad y eficiencia impuestos en el marco de las políticas evaluativas).

Para Bourdieu, el campo resulta de la configuración objetiva entre posiciones de actores o instituciones que intervienen en la lucha por la distribución del capital actual o potencial (o por los beneficios a obtener), y cuyo funcionamiento se define en tanto "haya algo en juego y gente que esté dispuesta a jugar, que esté dotada de los habitus que implican el conocimiento y reconocimiento de las leyes inmanentes al juego, de lo que está en juego, etcétera» (I990: I36). En tanto producto histórico, cada campo adquiere particularidad conforme a las fuerzas implicadas e intereses comunes; es decir, se distingue por el interés en monopolizar el capital específico ${ }^{11}$ dispuesto y acumulado en tanto fundamento de autoridad en ese campo, así como por el despliegue de estrategias para la conservación o subversión (conservación o transformación) de la configuración de fuerzas existentes (Bourdieu, I990; Bourdieu y Wacquant, 2005).

Como señalan Bourdieu y Wacquant, «el campo subyace y guía a las estrategias mediante las cuales los ocupantes de dichas posiciones buscan, individual o colectivamente, salvaguardar o mejorar su posición e imponer principios de jerarquización más favorable para sus propios productos» (2005: I 55 ). Las estrategias referidas dependen de la posición de los actores en el campo -distribución del capital específico- y de la percepción del campo según el punto de vista que se adopta desde la posición particular en la cual se sitúan. Desde esta perspectiva, todos los actores comprometidos en un campo están vinculados, conformando ese microcosmos debido a que poseen intereses fundamentales comunes (lo vinculado a la existencia del campo). Por ello, los antagonismos y luchas solapan la complicidad objetiva que supone el acuerdo sobre lo que se disputa -valor de lo que está en juego-, y que motiva a entrar en el juego aceptando implícita o explícitamente ciertas reglas y ciertos supuestos referidos al funcionamiento del campo; no se pone en entredicho el fundamento, axiomática fundamental, o el zócalo de creencias últimas sobre las cuales reposa el juego (Bourdieu, I990).

El campo implica los conceptos de capital y habitus, y que se entienden en

I I. Es el capital que juega en un campo determinado, dentro de los límites de ese campo, y sólo convertible en otra especie de capital bajo determinadas condiciones (Bourdieu, I990). 
el marco de los Bourdieu denomina filosofía disposicional, la «que toma en consideración las potencialidades inscritas en el cuerpo de los agentes y en la estructura de las situaciones en las que éstos actúan o, con mayor exactitud, en su relación» (1997: 7). En el campo los actores ocupan posiciones respecto del capital que poseen — recursos diferenciados para entrar en el juego-, buscando disputar, conservar o modificar la distribución desigual de poder derivado de ese capital. Se identifican tres tipos de capital: el capital económico (recursos monetarios y financieros), capital social (recursos entendidos como conjuntos de relaciones a ser movilizados por actores adscritos a redes y organizaciones, siendo individual, familiar o colectivo), y el capital cultural (disposiciones y hábitos adquiridos en la socialización como saberes, performances educativas, habilidades, así como formado por categorías de percepción y juicio). Además, el capital cultural puede aparecer en tres estados: objetivado (libros, hemerográficas, bases de datos, pinturas, música, susceptibles de ser heredados), subjetivado (consumo, apropiación, conocimiento, interiorización de la cultura incorporada por la trayectoria) e institucionalizado (títulos, constancias, diplomas, certificación independiente de lo que se ha incorporado). ${ }^{12}$

La densidad de lo social está dada por la trama de relaciones que configuran los diversos actores que disputan el capital, y por el hecho que ningún capital se construye de manera independiente, pudiendo producir cada uno un efecto sobre los otros. Cada campo señala las formas en que se valora, produce, transmite y conserva su capital, y es por ello que bienes, relaciones o saberes adquieren valor —o más valor- sólo en ciertos campos.

Si los campos corresponden a las estructuras objetivas, los habitus serían las estructuras incorporadas por la permanencia en un campo determinado. Actúan como organizadores/generadores de prácticas y representaciones en tanto

I2. Además, Bourdieu habla del capital simbólico, capital 'parasitario' que asume «cualquier propiedad (cualquier tipo de capital, físico, económico, cultural, social) cuando es percibida por agentes sociales cuyas categorías de percepción son de tal naturaleza que les permiten conocerla (distinguirla) y reconocerla, conferirle algún valor... ésa es la forma que adquiere cualquier tipo de capital cuando es percibido a través de unas categorías de percepción que son fruto de la incorporación de las divisiones o de las oposiciones inscritas en la estructura de la distribución de esta especie de capital (por ejemplo fuerte/débil, grande/ pequeño, rico/pobre, culto/inculto, etcétera)»( I997: 108). 
sistema de disposiciones adquiridas de manera implícita o explícita por medio del aprendizaje (Bourdieu, I990; I 997). ${ }^{13}$ En relación a los campos, el habitus otorga sentido de juego, es decir, implica tener el juego metido en la piel:

dominar en estado práctico el futuro del juego... tener el sentido de la historia del juego... [llevar] las tendencias inmanentes del juego en el cuerpo, en estado incorporado: formar cuerpo con el juego... es un cuerpo socializado, un cuerpo estructurado, un cuerpo que se ha incorporado a las estructuras inmanentes de un mundo o de un sector particular de este mundo, de un campo, y que estructura la percepción de este mundo y también la acción en este mundo (I997: I46).

De este modo, en el habitus lo social se interioriza en los actores, posibilitando la concordancia entre estructuras objetivas y subjetivas (Bourdieu I990), ${ }^{14}$ es decir, tiene lugar la negociación respecto de la producción y reproducción de sentido, siendo el punto donde confluyen lo estable, instituido, social (estructura objetiva) y lo emergente, instituyente, sociable (agencia). Por medio del habitus el actor se implica con el mundo, y actúa con sentido práctico; las actualizaciones derivadas de la experiencia cotidiana pasan inadvertidas, y son perceptibles en tanto perspectivas, valoraciones y acciones concretas, es decir,

I3. Bourdieu (2007) los definen como «sistemas de disposiciones duraderas y transferibles, estructuras estructuradas predispuestas a funcionar como estructuras estructurantes, es decir, como principios generadores y organizadores de prácticas y de representaciones que pueden ser objetivamente adaptadas a su meta sin suponer el propósito consciente de ciertos fines ni el dominio expreso de las operaciones necesarias para alcanzarlos, objetivamente 'reguladas' y 'regulares' sin ser para nada el producto de la obediencia a determinadas reglas, y, por todo ello, colectivamente orquestadas sin ser el producto de la acción organizadora de un director de orquesta» (86).

I4. Para Bourdieu (I997), «los habitus son principios generadores de prácticas distintas y distintivas - lo que come el obrero y sobre todo su forma de comerlo, el deporte que practica y su manera de practicarlo, sus opiniones políticas y su manera de expresarlas difieren sistemáticamente de lo que consume o de las actividades correspondientes del empresario industrial—; pero también son esquemas clasificatorios, principios de clasificación, principios de visión y de división, aficiones, diferentes. Establecen diferencias entre lo que es bueno y lo que es malo, entre lo que está bien y lo que está mal, entre lo que es distinguido y lo que es vulgar, etcétera, pero no son las mismas diferencias para unos y otros» (20). 
como resultado del sentido práctico. Las prácticas sociales están empotradas en las disposiciones alojadas en los actores y en las formas simbólicas que se han naturalizado, de manera que en la acumulación — desigual- de capital, lo actores se relacionan con las historia de sus prácticas y las prácticas que han incorporado en tanto representación, generando así esquemas de práctica diferenciados y diferenciadores. El habitus se expresa en el reconocimiento de formas concretas y categorizaciones de la realidad, en tanto esquemas de percepción, apreciación y acción por medio de los cuales el actor — desde su posición en el campo- otorga y articula sentido. A través de las clasificaciones, los actores se sitúan, dando cuenta de su posición social, y por tanto de su identificación/distinción con ciertos grupos sociales. Esto se encuentra mediado por el acceso desigual a ciertos tipos de capital, lo que gatilla diferentes visiones de la realidad y por ende refuerza relaciones de pertenencia o de identidad con ciertos actores o grupos de actores.

La confluencia entre habitus y la oferta de un campo constituye una base para el establecimiento de relaciones en el marco de una estrategia, es decir, distintas variantes a seguir en el juego. En tanto depositadas en el habitus, se expresan en trayectorias y orientaciones que se plasman en el posicionamiento del actor al interior del campo. Estas variantes se vinculan con la especialización de habitus, que implica la disposición para visualizar las exigencias para jugar en un campo concreto, y de ello, las estrategias para establecer relaciones y apropiar el capital específico del campo.

Con atención a lo anterior, habría que plantear las siguientes preguntas: ¿cómo se delimita un campo?, y ¿cómo se analiza un campo? Respecto a la primera, los límites del campo no admite presuposición, pues éstos se formulan dentro del campo mismo, es decir, desde la investigación. Por otro lado, la inclusión/exclusión de un actor respecto de un campo se define en la lucha por establecer criterios y principios de entrada y éxito en éste — exhibe los efectos de campo-, haciendo imposible explicar sus propiedades por características intrínsecas (Bourdieu y Wacquant, 2005). Respecto a la segunda, Bourdieu ha establecido procedimientos analíticos para abordar el estudio de los campos (Bourdieu y Wacquant, 2005: I60): a) analizar la posición del campo en el campo de poder, donde ocupa una posición dominada; b) establecer la estructura objetiva de las relaciones entre posiciones ocupadas por los agentes o instituciones que están en competencia en ese campo; c) analizar los habitus de los agentes, los diferentes sistemas de disposición que han adquirido al internalizar un determinado tipo de condición social y económica, condición 
que encuentra en su trayectoria del campo mayores o menores posibilidades de actualización.

En relación al proceder aclara que, aun cuando las posiciones objetivas (sistema estructurado de prácticas) y posturas (expresiones de los agentes) son metodológicamente inseparables, «es el campo el que primariamente es y debe ser foco de las operaciones de investigación» (I992 : I63). Esto no implica que los individuos sean mera ilusión, sino que son agentes, es decir, se encuentran socialmente constituidos en tanto activos y actuantes en el campo, y como poseedores de propiedades necesarias para ser efectivos y producir efectos en él.

\section{Teoría del campo y el campo universitario}

Bourdieu (2008) ha demostrado el potencial de la 'teoría del los campos' en su trabajo Homo academicus, investigación iniciada a mediados de los sesenta, y que versa sobre diversas facultades de universidades o grandes escuelas en el marco de lo que denominó campo universitario, vinculando la dinámica interna (autonomía relativa del campo universitario) con elementos externos (campo político, campo social) en un análisis relacional e integrador.

El campo universitario, entendido como ese juego de relaciones objetivas entre varias posiciones y disciplinas en la distribución de estas especies de capital, se constituye en lugar de pugnas constantes que se orientan a la transformación de la estructura. El poder académico y el prestigio intelectual son los elementos esgrimidos en la lucha, en tanto que la posición en la estructura define, por selección y condicionamiento específico, las estrategias adoptadas para imponerse como principio de la jerarquización dentro del universo específico. Más allá de las dinámicas propias del campo universitario —en el sentido de campo relativamente autónomo-, Bourdieu desentraña la lógica de su transformación, particularmente de aquellas derivadas de cambios morfológicos. Entre ellos, el que más destaca es el incremento de estudiantes que define el crecimiento desigual de los cuerpos docentes, y, por otro lado, la transformación de la relación de fuerzas entre facultades y disciplinas en su interior. Esto le permite establecer una homología entre la posición (en la estructura de la distribución de varias especies de capital en el campo universitario) y la disposición, las que no sólo operan en las prácticas profesionales de diferente tipos de académicos, sino que se orientan explícitamente desde lo político tiñendo opiniones y acciones tomadas durante un período de crisis.

Lo que define la organización del campo universitario refiere a dos prin- 
cipios de jerarquización antagónicos: a) jerarquía social conforme al capital heredado, capital económico y capital político, b) jerarquía cultural (capital de autoridad científica o notoriedad intelectual). Estos principios de jerarquización conforman, a su vez, dos principios de legitimación en pugna: uno de carácter temporal y político, y otro fundado en la autonomía de orden científico e intelectual.

De este modo, la estructura y volumen de los capitales que permiten situar a los actores en diferentes posiciones en el campo (dominantes/dominados) remite a (Bourdieu, 2008:59-60): a) índole social (posibilidades de acceso a las posiciones ocupadas, es decir, el capital económico, político y social heredado, el origen social — profesión del padre, prestigio social, origen geográfico y la religión de la familia de origen-); b) capital académico (éxito académico durante los estudios secundarios y superiores y los títulos obtenidos); c) capital de poder universitario (posiciones ocupadas en la jerarquía institucional como decano, director de una unidad de docencia e investigación, pertenencia a jurados, etcétera); d) capital del poder científico (dirección de un organismo de investigación, participación en una revista científica, participación en un consejo superior de investigación); e) capital de prestigio científico (distinciones científicas, traducciones en lenguas extranjeras, participación en coloquios internacionales, citación en índex de revistas); f) capital de notoriedad intelectual (pertenencia a la Academia Francesa y mención en Larousse, apariciones en televisión, colaboración en diarios o semanarios, revistas intelectuales, pertenencia a comités de redacción de revistas intelectuales); c) capital de poder político o económico (pertenencia a gabinetes ministeriales, enseñanza en las écoles de poder, condecoraciones diversas); d) de suposiciones políticas (participación en coloquios de Caen y Amiens, firma de peticiones diversas)..$^{15}$

Otro aporte del autor se relaciona con la distinción que establece respecto a la posición de ciertas facultades y carreras universitarias. Para Bourdieu

I 5. Más allá que el sistema universitario francés sea muy distinto al de muchos países latinoamericanos, quienes jugamos en el campo universitario en nuestro rol académico podemos reconocer fácilmente como estos capitales están presentes. Podemos mencionar: si estudió una maestría o doctorado en Estados Unidos o Europa versus si estudió en América Latina o en su país; si la institución estuvo dentro de las primeras Ioo del ranking; si publica en una revista ISI QI, Q4 o una de corriente principal; si publica en inglés-lengua extranjera o en la lengua nativa; si participa en congresos internacionales o nacionales; etcétera. 
(2008), existen facultades que comprenden el polo mundano (derecho y medicina) y el polo científico (ciencias y letras), en tanto "sitio privilegiado para observar la lucha entre las dos especies de poderes universitarios que, en los dos polos del campo, tienden a imponerse casi exclusivamente» (I7I). En medicina y derecho, el poder universitario (cúmulo de posiciones que permiten controlar otras posiciones y ocupantes) está tan afirmado, que investigadores puros o fundamentalistas son desplazados y remitidos a otro orden, como el de la facultad de ciencias, donde tienden a ser menos reconocidos que los científicos puros. Para la facultad de ciencias, el prestigio científico, que se funda en la inversión exitosa en la actividad excluyente de investigación, «tiende a hacer aparecer como sustituto compensatorio el poder dominado que, en el lugar mismo del no poder, ejercen los rectores, decanos y otros administradores científicos (aunque siempre se pueda oponer algunos casos de administradores reconocidos científicamente)». En las facultades de letras y ciencias humanas, las relaciones entre los diferentes principios de jerarquización están más equilibradas, en tanto participan del campo científico y del campo intelectual (la notoriedad intelectual), y, por el otro, en tanto lugar de poderes propiamente sociales, esto al ser la institución referida a consagrada y conservar la transmisión de la cultura legítima. ${ }^{16}$

Considerando la transformación global que ha vivido el sistema de educación superior y con él la universidad desde los años ochenta, sin duda muchas de las interpretaciones realizadas por Bourdieu pueden ser sometidas a revisión. Resulta válido preguntar acerca de la existencia y características de un campo universitario, del tipo de capital que allí se pone en juego, legitima y otorga el dominio de unos agentes sobre el campo, y sobre qué estrategias se despliegan en dicho campo, entre otras. Sin embargo, más allá de la antigüedad y el 'localismo' de la obra que caracteriza al 'homo academicus gallicus', el mismo Bourdieu señala (Bourdieu y Wacquant, 2005) que su propuesta puede ser tomada como un programa de investigación de cualquier campo académico, y por trasposición

descubrir, por razonamiento nomológico, un buen número de cosas

I6. Bourdieu señala que en las facultades de letras y de ciencias humanas se homologa la lucha que se establece en el seno del campo universitario en su conjunto entre las facultades dominantes en el orden cultural (intelectual) y las facultades dominantes en el orden propiamente temporal. 
sobre su propio universo profesional [bajo la hipótesis], que encontraríamos las mismas oposiciones fundamentales, en particular entre el capital académico vinculado al poder sobre los instrumentos de reproducción y el capital intelectual vinculado al renombre científico, pero tal oposición aparecería expresada de forma distinta (I22-I23).

\section{¿Un campo, varios campos? Algunas reflexiones}

Entonces, ¿es probable que exista o sea posible de aplicar la 'teoría del campo', y por consiguiente hablar de un campo universitario en el contexto latinoamericano?

Pedro Krotsch (2009) señaló que resulta dudoso para el caso argentino «en tanto no existen aún reglas del juego que aludan a formas de distribuir prestigio entre distintos modos de investigar o maneras de regular el acceso y pertenencia al mismo» (65-66), agregando que las tensiones son difusas, y que existe un importante aislamiento entre las distintas posiciones que ocupan los actores en el sistema y las instituciones.

Sin embargo, no se puede desconocer que la universidad constituye un espacio donde entra en juego: la producción y difusión de conocimiento, actores (académicos, estudiantes) dispuestos a jugar el juego y que reafirman sus creencia en los sistemas de recompensas y reconocimientos en tanto base de posiciones y disposiciones para el desenvolvimiento, criterios de inclusión/exclusión que dinamizan la disputan respecto de lo que debe operar en el campo (por ejemplo: ciencia/profesión, investigación/docencia, autonomía/heteronimia). La transformación de la universidad puede ser definida ella misma como el resultado de las luchas en el campo universitario, en tanto se busca zanjar el capital específico y su apropiación. Además, es posible visualizar intereses comunes entre los actores y la complicidad básica que involucra aceptar las reglas del juego, las que son delimitadas en torno a 'lo académico' en tanto locus de investigación, formación y difusión de cultura y conocimiento especializado.

También sería posible distinguir el capital cultural como definitorio en tanto supone: a) socialización y trayectoria —experiencia y saber acumulado- en un área del conocimiento — disciplina-, habilidades comunicativas ligadas a la enseñanza, a la comunicación, etcétera; b) existencia de objetos y tecnologías en tanto recursos y medios (laboratorios, salas, bibliotecas, etcétera); c) títulos, diplomas y reconocimientos institucionalizados en la universidad, que 
se confieren se requiere (para los académicos). Cobra además importancia el capital ligado a lo social (vínculos con corporaciones y sociedades académicas, redes o núcleos de investigación) y simbólico (vinculado al prestigio, reconocimiento en la jerarquía y legitimidad).

Por otro lado, los mismos Estados están promoviendo ciertas reglas de juego en el marco del modelo de 'estado evaluador' (Brunner, I990; Araujo, 2007; Chiroleu, 2007a). Se han establecido criterios de calidad asociados a lo que se denomina acreditación institucional (opera en gestión institucional, docencia de pregrado y posgrado, investigación, vinculación con el medio), acreditación de carreras y programas de posgrado, ${ }^{17}$ todo lo que se acompaña de recursos y prestigio (bajo la forma de capital simbólico, capital académico, capital científico y económico). Lo anterior, además, ha repercutido en la implementación de la jerarquización a través de categorizaciones y evaluaciones del desempeño docente (docencia, investigación, extensión), afectando las remuneraciones y estatus académico (jerarquización). Lo que sucede en la trastienda de la acreditación institucional y la jerarquización académica es sin duda estimulante de analizar desde la 'teoría de los campos', pudiendo señalar que marcan en gran medida la dinámica del funcionamiento del 'campo universitario'.

Se podría hipotetizar que en el nuevo contexto de la educación superior universitaria, el capital académico, que podría pensarse como definitorio del campo, subordina cada vez más su reproducción al capital económico y político (financiamiento para investigaciones, publicaciones, programas formativos), y por tanto a quienes deciden (campo político o propiamente económico), cuestión que, a diferencia de lo planteado por Bourdieu (2008), podría ser transversal para todas las facultades.

Lo central podría estar cambiando, tal y como señalan algunos estudios sobre educación superior, entrando con fuerza definiciones de mercado ligadas a la optimización de recursos que muestra su faceta en la redefinición curricular y los perfiles profesionalizantes, y las exigencias de máxima productividad académica (producción de proyectos, papers, patentamientos, etcétera) en desmedro de aquellos de orientación científica tradicional.

En la lógica de la universidad-empresa, el capital económico se reconvierte en capital simbólico, en tanto constituye fuente de prestigio y de éxito (jerarquía) — por ejemplo, a partir de la obtención de patentes, de negocios, etcétera- cada vez que implican la captura de financiamiento privado a la universi-

I7. Véase <http://www.cnachile.cl/materiales/materiales.html>. 
dad. Una hipótesis al respecto puede ser que la estrategia del actor-académico en este nuevo contexto —-donde el campo se ha invertido-, sea reducir la violencia simbólica apelando a la excelencia científica, de manera de no perder las cuotas de decisión sobre lo que es y no es pertinente en el campo, apelando además a la transparencia y democratización de las prácticas académicas. ${ }^{18} \mathrm{En}$ este sentido, se generaría un fenómeno similar al que Bourdieu (I990) describe para los campos de producción de bienes culturales: «la subversión herética afirma ser un retorno a los orígenes, al espíritu, a la verdad del juego, en contra de la banalización y degradación de que ha sido objeto» (I I I ). ${ }^{19}$

Por otro lado, no se puede desconocer la complejidad alcanzada por el 'sistema universitario', donde confluyen diversos actores-instituciones (gobierno universitario, empresa privada, Estado, gobierno local y los denominados clientes), misma diversidad que es posible de encontrar en su 'interior' entre actores que gobiernan (rectores, directores generales, decanos, etcétera), administrativos (vinculados a servicios), académicos (docentes tiempo completo con contrato de planta, tiempo parcial con contrato de planta, part-time o freelance) y trabajadores (auxiliares, jardineros, etcétera). Lo anterior llevaría a plantear la interrogante acerca de qué está dentro y qué está fuera del campo universitario (como opera la autonomía relativa), obligándonos a redefinir cuáles son los actores que allí concurren propios del campo-. En esta

I 8. Incluso se puede llegar a discutir la idea de que las diferentes disciplinas y facultades (o departamentos) constituyen configuraciones específicas de intereses, lo que queda reflejado en la convergencia de actividades, y por tanto unificar las formas de pensar las tareas académicas, las funciones de la universidad, y los criterios de jerarquización.

19. Al respecto, Bourdieu plantea que «aquellos que, dentro de un estado determinado de la relación de fuerzas, monopolizan (de manera más a menos completa) el capital específico, que es el fundamento del poder de la autoridad especifica característica de un campo, se inclinan hacia estrategias de conservación -las que, dentro de los campos de producción de bienes culturales, tienden a defender la ortodoxia-, mientras que los que disponen de menos capital (que suelen ser también los recién llegados, es decir, por lo general, los más jóvenes) se inclinan a utilizar estrategias de subversión: las de la herejía. La herejía, la heterodoxia, como ruptura critica, que está a menudo ligada a la crisis, junta con la doxa, es la que obliga a los dominantes a salir de su silencio y les impone la obligación de producir el discurso defensivo de la ortodoxia, un pensamiento derecho y de derechas que trata de restaurar un equivalente de la adhesión silenciosa de la doxa» (I990: I37). 
dirección, se debería revisar la discusión entre autonomía-heteronomía universitaria a la luz de un abordaje como el propuesto por Bourdieu, y ver si esta disputa tiene como trasfondo la lucha entre el polo 'mundano' y 'científico' por la delimitación y definición del campo.

Dicha complejidad requiere la posibilidad de jugar con niveles de análisis, pudiendo una aproximación 'macro' abordar el 'campo universitario' considerando como actores a las universidades públicas y privadas que se diputan el capital simbólico y económico, analizar cómo juegan -se posicionan y actúan- las facultades, disciplinas y académicos, y visualizar como entra en juego la empresa privada y la política de gobierno. ${ }^{20}$ A nivel 'micro,' es posible situar el análisis en el 'campo universitario' como referido a una universidad particular, o a un subcampo -dimensión. Aquí se podría mencionar: a) el 'campo académico' — posible lucha por el capital académico-, pudiendo definir el peso relativo que juegan otros tipos de capital — como el 'capital social y cultural heredados', el 'capital docente', el 'capital investigador o científico', el 'capital de prestigio' (dado por la participación en sociedades y comités), el 'capital de visibilidad' (dado por la extensión y notoriedad pública)— para establecer criterios de inclusión/exclusión y jerarquización, o de otro modo, configurando otros campos—como por ejemplo, el campo docentes para las universidades que no enfatizan la investigación-; b) el 'campo profesional' -posible lucha por el capital económico- donde intervienen sindicatos, sociedades, trabajadores y gobierno universitario.

Si un campo se configura en tanto se dispute la hegemonía y control del capital específico puesto en juego, así como requiere de criterios de inclusión/ exclusión para su funcionamiento, los ejemplos brevemente expuestos hacen pensar en la posibilidad de aplicación de esta teoría para el contexto latinoamericano. Se reconoce en la 'teoría de los campos' el potencial analítico para explicar, en distintos niveles o en su conjunto, las dinámicas que atraviesan a la universidad, distinguiendo como entran en escena condicionantes internas (gobierno universitario, facultades, disciplinas, académicos, estudiantes) y ex-

20. Se establece una lucha por 'los abundantes clientes' que se grafica en alcanzar mayores cuotas de matriculados, y pone en juego estrategias para este logro mediante el marketing y la diferenciación (por ejemplo universidades que han incorporado el modelo por competencia, o que apuntan al fortalecimiento de un área de conocimiento o facultad). Por otro lado, estudiar en tal o cual universidad aumenta el volumen del capital social y cultural del estudiante y futuro ingresante al mercado laboral. 
ternas o de contexto (Estado, mercado, empresa privada, etcétera). Lo que se plantea en el cierre de este trabajo, es que la delimitación de un campo esta mediada por la escala de análisis que utiliza el investigador, y que, por tanto, jugar con el foco posibilita identificar en el cosmos, microcosmos o campos y subcampos.

\section{Referencias}

Alcántara, A. (2000). «Tendencias mundiales en la educación superior. El papel de los organismos multilaterales». En D. Cazés, L. Porter y E. Ibarra (coords), Reconociendo a la universidad: sus transformaciones y su porvenir (pp. 8I-IO2). (Tomo I. Estado, Universidad y Sociedad: entre la globalización y la democratización). México: CEIICH-UNAM.

Altbach, Ph. (200I). La educación superior comparada. El conocimiento, la universidad y el desarrollo. Cátedra UNESCO de Historia y Futuro de la Universidad. Buenos Aires: Universidad de Palermo.

-. (2003). «Centers and Peripheries in Academic Profession: The Special Challenges of Developing Countries». En Ph. Altbach (ed.), The Decline of the Guru.The Academic Profession in the Third World (pp. I-2I). Nueva York: Palgrave MacMillan.

- (2008). "Funciones complejas de las universidades en la era de la globalización». En La educación superior en el mundo 2008. La financiación de las universidades. Disponible en <http://upcommons.upc.edu/revistes/bitstream/2099/793 I/I/O2\% 20(5-I4).pdf>.

Araujo, S. (2007). «Evaluación institucional y cambio universitario. Un difícil proceso de reconstrucción». En P. Krotsch, A. Camou y M. Prati (coords.), Evaluando la evaluación. Politicas universitarias, instituciones y actores en Argentina y América Latina (pp. 69-94). Buenos Aires: Prometeo.

Ben DAvid, J. y Zloczower (I966). «Universidades y sistemas académicos en las sociedades modernas». En A. H. Halsey, A. Raymond, M. Trow y R. Dahrendorf, La universidad en transformación. Barcelona: Seix-Barral.

Bourdieu, P. (2007). Sentido práctico. Buenos Aires: Siglo XXI.

-. (2008). Homo academicus. Buenos Aires: Siglo XXI.

-. (I990). Sociología y cultura. México: Grijalbo.

-. (I997). Razones prácticas. Sobre la teoría de la acción. Barcelona: Anagrama.

-. (2008). Los usos sociales de la ciencia. Buenos Aires: Nueva Visión.

-. (2006). Intelectuales, política y poder. Buenos Aires: Eudeba. 
Bourdieu, P. y L. WACQUANT (2005). Una invitación a la sociología reflexiva. Buenos Aires: Siglo XXI.

BrunNer, J. (2007). Universidad y sociedad en América Latina: La sociología de una ilusión moderna. Caracas: CRESALC-ILDIS.

-. (I990). La educación superior en América Latina: cambios y desafíos. Santiago: Fondo de Cultura Económica.

-. (I997). «Educación superior, integración económica y globalización». Perfiles Educativos, tercera época, I9 (76-77): 6-I 5.

Clark, B. (I992). El sistema de educación superior. México: Nueva Imagen.

Chiroleu, A. (2007a). «Según pasan los años: de la autonomía universitaria y sus retos». En E. Rinesi y G. Soprano (eds.), Facultades alteradas. Actualidad del conflicto de facultades de Kant (pp. I I9-I43). Buenos Aires: Prometeo.

-. (2007b). «Los contextos que enmarcan a reforma de la ley de educación superior: entre desafíos y oportunidades». En M. Marquina y G. Soprano (coords), Ideas sobre la cuestión universitaria. Aportes de la RIEPESAL al debate sobre el nuevo marco legal para la Educación Superior (pp. 37-56). Buenos Aires: Universidad Nacional General Sarmiento.

Chiroleu, A. y O. Iazzetta (2005). «La reforma de la educación superior como capítulo de la reforma del Estado. Peculiaridades y trazos comunes». En E. Rinesi, G. Soprano y C. Suasnábar (comp.), Universidad: reformas y desafíos. Dilemas de la educación superior en la Argentina y el Brasil (pp. I 5-38). Buenos Aires: Prometeo.

Chiroleu, A. y L. Vilosio (2007). «Los organismos internacionales y la educación superior. Recomendaciones y propuestas para el siglo XXI». En M. Marquina y G. Soprano (coords), Ideas sobre la cuestión universitaria. Aportes de la RIEPESAL al debate sobre el nuevo marco legal para la Educación Superior (pp. 57-74). Buenos Aires: Universidad Nacional General Sarmiento.

García, C. (2003). "Balance de la década de los noventa y reflexiones sobre las nuevas fuerzas de cambio en la educación superior». En M. Mollis (comp.), Las universidades en América Latina: ¿reformadas o alteradas? La cosmética del poder financiero (pp. I7-37). Buenos Aires: Clacso.

-. (2006). «Complejidades de la globalización y la comercialización de la educación superior. Reflexiones para el caso de América Latina». En H. Vessuri, Universidad e investigación científica (pp. I35-I68). Buenos Aires: Clacso. 
GonzÁlez, L. (2003). "Acreditación y fomento de la calidad. La experiencia chilena en las últimas décadas». En M. Mollis (comp.), Las universidades en América Latina: ¿reformadas o alteradas? La cosmética del poder financiero (pp. I4I-I 59). Buenos Aires: Clacso.

Ibarra Colado, E. (2005). "Origen de la empresarialización de la universidad: el pasado de la gestión de los negocios en el presente del manejo de la universidad». Revista de Educación Superior, 34 (2): 13-37.

Krotsch, P. (2009). Educación superior y reformas comparadas. Buenos Aires: Universidad Nacional de Quilmes.

LEVY, D. (I994). Introducción. En D. Levy. (coord.), La educación superior dentro de las transformaciones políticas y económicas de los años noventa. Informe del grupo de trabajo sobre educación superior de la asociación de estudios latinoamericanos. Buenos Aires: CEDES, Argentina. Disponible en $<$ http://bibliotecavirtual.clacso.org.ar/ar/libros/argentina/cedes/levy.rtf>.

López, F. (2003). «El impacto de la globalización y las políticas educativas en los sistemas de educación superior de América Latina y el Caribe». En M. Mollis (comp.), Las universidades en América Latina: ¿reformadas o alteradas? La cosmética del poder financiero (pp. 39-58). Buenos Aires: Clacso.

Llomovatte, S. (2006). «Para una crítica del modelo de la triple hélice: universidad, empresa y Estado». En S. Lomovatte, F. Juarros, J. Naidorf y A. Guelman, La vinculación universidad-empresa: una crítica desde la universidad pública. Buenos Aires: Miño y Dávila Editores.

Marquina, M. (2007). «El conflicto de la profesión académica: entre la autonomía, la burocratización y la mercantilización». En E. Rinesi y G. Soprano (eds.), Facultades alteradas. Actualidad del conflicto de facultades de Kant (pp. I45-I73). Buenos Aires: Prometeo.

Mazzola, C. (2007). "La evaluación y la autonomía universitaria». En M. Marquina y G. Soprano (coords), Ideas sobre la cuestión universitaria. Aportes de la RIEPESAL al debate sobre el nuevo marco legal para la Educación Superior (pp. 77-88). Buenos Aires: Universidad Nacional General Sarmiento.

Mollis, M. (2003). Presentación. En M. Mollis (comp.), Las universidades en América Latina: ¿reformadas o alteradas? La cosmética del poder financiero (pp. 9-I 5). Buenos Aires: Clacso.

-. (2006). «Geopolítica del saber: biografías recientes de universidades latinoamericanas». En H. Vessuri, Universidad e investigación científica (pp. 85-IOI) Buenos Aires: Clacso. 
Muñoz, H. y R. Rodríguez (2000). «Educación superior en México. Diferenciación y cambio hacia el fin de siglo». En H. Casanova y otros, Diversidad y convergencia. Estrategias de financiamiento, gestión y reforma de la educación superior (pp.I27-I 50). México: UNAM/CESU.

Prati, M. y C. Prego (2007). "Cultura académica y producción de conocimiento en el marco de las políticas de incentivos. Un enfoque comparado de ciencias básicas y humanidades». En P. Krotsch, A. Camou, y M. Prati (coords.), Evaluando la evaluación. Políticas universitarias, instituciones y actores en Argentina y América Latina (pp. 267-308). Buenos Aires: Prometeo.

Rinesi, E. y G. Soprano (2007). «Universidad, Estado y sociedad. Los sentidos de la autonomía y la heteronomía en la experiencia de la universidad pública en Argentina». En M. Marquina y G. Soprano (coords), Ideas sobre la cuestión universitaria. Aportes de la RIEPESAL al debate sobre el nuevo marco legal para la Educación Superior (pp. I 5-24). Buenos Aires: Universidad Nacional General Sarmiento.

Rodríguez, R. (2003). "La educación superior en el mercado. Configuraciones emergentes y nuevos proveedores». En Marcela Mollis (ed.), Las universidades en América Latina. ¿Reformadas o alteradas? (pp. 87-107) Buenos Aires: Clacso.

Rodríguez, R. y A. Alcántara (2003). «Toward a Unified Agenda for Change in Latin American Higher Education. The Role of Multilateral Agencies». En S. J. Ball, G. Fischman y S. Gvirtz (eds.), Crisis and Hope. The Educational Hopscotch of Latin America (pp. 19-44). Nueva York: Routledge and Falmer.

SChugurensky, D. (I998). «Reestructuración de la educación superior en la era de la globalización: ¿hacia un modelo heterónomo?» En A. Alcántara, R. Pozas y C. Torres (coords), Educación, democracia y desarrollo en el fin de siglo (pp. 59-78). México: Siglo XXI.

-. (2000). "Autonomía, heteronomía y los dilemas de la educación superior en la transición al siglo 2 I; caso Canadá». En R. Rodríguez, (coord.), Reformas en los sistemas nacionales de educación superior. Coruña: Netbilblo.

Tünnermann, C. (2003). La universidad latinoamericana ante los retos del siglo XXI. México: Unión de Universidades Latinoamericanas. 


\section{Sobre el autor}

Héctor Mora Nawrath es licenciado en Antropología, magíster en Ciencias Sociales y doctorando en Ciencias Sociales. Es director de la carrera de Antropología de la Universidad Católica de Temuco. Su correo electrónico es $<$ hectmora@uct.cl>. 